\title{
Application of the DSM-5 Criteria for Major Neurocognitive Disorder to Vascular MCI Patients
}

\author{
Emilia Salvadori $^{a} \quad$ Anna Poggesi ${ }^{a} \quad$ Giovanni Pracucci $^{a} \quad$ Alberto Chiti $^{b}$ \\ Laura Ciolli $^{a} \quad$ Mirco Cosottini $^{c} \quad$ Alessandra Del Bene ${ }^{a}$ Nicola De Stefano ${ }^{d}$ \\ Stefano Diciotti ${ } \quad$ Ilaria Di Donato ${ }^{d}$ Andrea Ginestroni ${ }^{f}$ Sandro Marini ${ }^{a}$ \\ Mario Mascalchig, herena Nannucci ${ }^{a}$ Giovanni Orlandi ${ }^{b}$ \\ Marco Pasi ${ }^{a}$ Francesca Pescini ${ }^{i}$ Raffaella Valenti ${ }^{a}$ Antonio Federico ${ }^{d}$ \\ Maria Teresa Dotti ${ }^{d}$ Ubaldo Bonuccellib Domenico Inzitaria \\ Leonardo Pantonij on behalf of the VMCl-Tuscany Study Group \\ ${ }^{a}$ NEUROFARBA Department, Neuroscience Section, University of Florence, Florence, \\ Italy; ${ }^{b}$ Department of Clinical and Experimental Medicine, University of Pisa, Pisa, Italy; \\ 'Department of Translational Research and New Technologies in Medicine and Surgery, \\ University of Pisa, Pisa, Italy; ${ }^{d}$ Department of Medicine, Surgery, and Neurosciences,

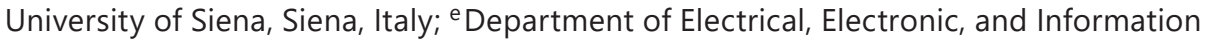 \\ Engineering "Guglielmo Marconi," University of Bologna, Cesena, Italy; ${ }^{\text {N Neuroradiology }}$

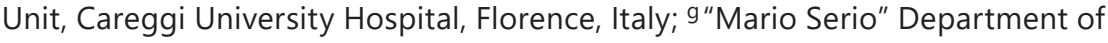 \\ Experimental and Clinical Biomedical Sciences, University of Florence, Florence, Italy; \\ ${ }^{\mathrm{h}}$ Quantitative and Functional Neuroradiology Research Program at Meyer Children Hospital

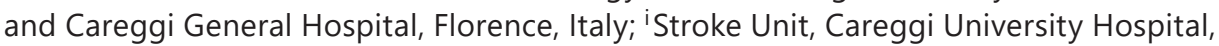 \\ Florence, Italy; " "L. Sacco" Department of Biomedical and Clinical Sciences, University of \\ Milan, Milan, Italy
}

Keywords

Small vessel disease - Mild cognitive impairment - Dementia - Cerebrovascular diseases · Neuropsychology

\section{Abstract}

Aims: The DSM-5 introduced the term "major neurocognitive disorders" (NCDs) to replace the previous term "dementia." However, psychometric and functional definitions of NCDs are missing. We aimed to apply the DSM-5 criteria for diagnosing the transition to NCD to patients with mild cognitive impairment $(\mathrm{MCl})$ and small vessel disease (SVD), and to define clinically significant thresholds for this transition. Methods: The functional and cognitive features of the NCD criteria were evaluated as change from baseline and operationalized according to 
hierarchically ordered psychometric rules. Results: According to the applied criteria, out of 138 patients, 44 were diagnosed with major NCD (21 with significant cognitive worsening in $\geq 1$ additional cognitive domain), 84 remained stable, and 10 reverted to normal. Singledomain $\mathrm{MCl}$ patients were the most likely to revert to normal, and none progressed to major NCD. The amnestic multiple-domain $\mathrm{MCl}$ patients had the highest rate of progression to NCD. Conclusion: We provide rules for the DSM-5 criteria for major NCD based on cognitive and functional changes over time, and define psychometric thresholds for clinically significant worsening to be used in longitudinal studies. According to these operationalized criteria, onethird of the $\mathrm{MCl}$ patients with SVD progressed to major NCD after 2 years, but only within the multiple-domain subtypes.

(c) 2018 The Author(s)

Published by S. Karger AG, Basel

\section{Introduction}

Dementia is a common outcome measure in several observational studies and clinical trials of mild cognitive impairment (MCI) patients. A dementia diagnosis is often based on standard and widely used criteria that are, however, poorly operationalized. Among the studies focused on dementia diagnosis there is a large heterogeneity in terms of the neuropsychological tests (focused either on global functioning or specific cognitive abilities), number and type of cognitive domains, and psychometric thresholds used to define objective impairment in neuropsychological test performance. This may introduce discrepancies in both research and clinical settings. Moreover, dementia criteria based on memory deficits, mainly developed for Alzheimer disease, may not be suitable for dementia associated with cerebrovascular diseases, in which memory dysfunctions could be less prominent $[1,2]$.

In 2013, the American Psychiatric Association published the 5th edition of the Diagnostic and Statistical Manual of Mental Disorders (DSM-5), which introduced the new term "neurocognitive disorders" (NCDs) to replace the DSM-IV category "delirium, dementia, amnestic and other geriatric cognitive disorders" [3]. In the DSM-5 criteria for NCDs, impairment in any cognitive domain, including executive function, is sufficient for the diagnosis, and the memory domain is no longer hierarchically prominent [3].

Another relevant change in the DSM-5 criteria is the distinction between "mild" and "major" NCDs, with the first term being closely aligned with MCI. The authors of the DSM-5 clearly state that mild and major NCDs exist along a continuum, and that precise thresholds are difficult to determine, but they recommend evaluating an individual's performance in light of the prior administration of the same test [3]. Measuring the magnitude of cognitive change over time is therefore of utmost importance in evaluating the transition from mild to major NCD. At present, however, criteria for defining clinically significant psychometric thresholds for change from a previous level are missing.

The main objective of the present study was to contribute to the emerging line of research focused on statistically derived neuropsychological criteria for the operational definition of cognitive impairment of various degrees. In the present study, we aimed at operationalizing the DSM-5 criteria for determining the transition to major NCD in a sample of MCI patients with cerebral small vessel disease (SVD) by means of (1) defining a set of hierarchically ordered psychometric thresholds for cognitive worsening from a previous level, and (2) evaluating how the application of progressively less severe thresholds for cognitive worsening could increase the rate of patients diagnosed with major NCD. We further aimed at assessing the outcomes of patients with MCI and SVD also in terms of the association between baseline $\mathrm{MCI}$ subtype and the transition to major NCD using the operationalized criteria. 


\section{Materials and Methods}

The Vascular MCI (VMCI)-Tuscany Study is a multicenter, prospective, observational study with the primary objective of examining the determinants of transition from VMCI with SVD to dementia. The study methodology has been reported in detail elsewhere [4]. The study was conducted in accordance with the Helsinki Declaration and was approved by the local ethics committee; each patient gave written informed consent.

To be included, patients had to be classified as (1) affected by MCI according to the criteria of Winblad et al. [5], and operationalized according to Salvadori et al. [6], and (2) showing evidence on MRI of moderate-to-severe white matter hyperintensities (WMHs) according to a modified version of the Fazekas scale [7]. The degree of WMH severity was rated on FLAIR (fluid-attenuated inversion recovery) sequences taking into account only deep and subcortical white matter lesions. The modified Fazekas scale is a visual scale based on a categorization into 3 severity classes: grade 1 (mild $\mathrm{WMH})=$ single lesions $<10 \mathrm{~mm}$, areas of "grouped" lesions $<20 \mathrm{~mm}$ in any diameter; grade 2 (moderate $\mathrm{WMH}$ ) = single lesions between 10 and $20 \mathrm{~mm}$, areas of "grouped" lesions $>20 \mathrm{~mm}$ in any diameter, no more than "connecting bridges" between individual lesions; and grade 3 (severe $\mathrm{WMH}$ ) = single lesions or confluent areas of hyperintensity $\geq 20 \mathrm{~mm}$ in any diameter. According to the study protocol, at baseline each patient underwent a comprehensive clinical, neuropsychological, and functional evaluation that was repeated after 12 and 24 months. The neuropsychological protocol, namely, the VMCI-Tuscany neuropsychological battery [8], and the functional scales $[9,10]$ are detailed in Table 1.

At baseline evaluation, the diagnosis of MCI required $\geq 1$ borderline score (an adjusted score between the outer and inner 95\% confidence limits for the 5 th percentile of the normal population according to published normative data) among the 12 scores deriving from the 9 neuropsychological tests included in the VMCI-Tuscany neuropsychological battery [8]. The MCI subtypes were further divided into amnestic (single or multiple domain) and nonamnestic (single or multiple domain) according to the criteria of Winblad et al. [5]. Despite the fact that the main outcome declared in the original VMCI-Tuscany Study protocol was transition from MCI to dementia diagnosed according to the DSM-IV criteria [4], we decided to apply the more recent DSM-5 criteria for major NCDs and to reformulate the VMCI-Tuscany Study's main outcome as transition from MCI to major NCD.

In applying the DSM-5 criteria in our longitudinal research setting, we introduced some methodological arrangements, detailed below, to ensure the harmonization of different needs: (1) a standardized diagnostic algorithm to guarantee reproducibility, and (2) a caseby-case evaluation of clinical status and of the occurrence of comorbidities that could influence the functional status in the elderly.

The diagnosis of major NCD was operationalized as follows. For each patient, we evaluated separately the 2 core features of the DSM- 5 criteria: evidence of a significant cognitive decline (cognitive outcome) and interference with the capacity for independence in everyday activities (functional outcome). Both cognitive and functional outcomes were defined as change compared to the baseline evaluation, and categorized as "improved," "stable," or "worsened."

Change in functional outcome was based on the Activities of Daily Living (ADL) [9] and Instrumental Activities of Daily Living (IADL) [10] scales, and the "worsened" condition was defined as the occurrence of at least one of 2 conditions: (1) loss of $\geq 1$ ADL item, i.e., the patient has become dependent in $\geq 1$ item function that was preserved at the baseline evaluation, and (2) loss of $\geq 2$ IADL items, i.e., the patient has become dependent in $\geq 2$ activities that were preserved at the baseline evaluation.

A worsened functional outcome was considered an indispensable prerequisite for transition to major NCD, and represented the first step in the operationalized diagnostic algo- 
Table 1. Neuropsychological and functional evaluation

\begin{tabular}{ll}
\hline Cognitive domain & Test \\
\hline $\begin{array}{l}\text { VMCI-Tuscany neuropsychological battery } \\
\text { Global mental functioning }\end{array}$ & Mini-Mental State Examination \\
Memory & Montreal Cognitive Assessment \\
& Rey Auditory Verbal Learning Test \\
& (immediate and delayed recall) \\
& Short story \\
& Rey-Osterrieth Complex Figure (recall) \\
Trail Making Test, parts A and B & Visual search \\
Attention/executive function & Symbol Digit Modalities Test \\
& Stroop Color-Word Test \\
& Phonemic verbal fluency \\
& Semantic verbal fluency \\
Language & Rey-Osterrieth Complex Figure (copy) \\
Visuoconstructional abilities & Activities of Daily Living scale \\
\hline Functional evaluation & Instrumental Activities of Daily Living scale \\
\end{tabular}

rithm. As a second step, the changes in cognitive outcome were evaluated. To this purpose, based on the VMCI-Tuscany neuropsychological battery, we considered changes in performance on the 12 scores deriving from the 9 second-level tests (Table 1), excluding the global cognitive functioning ones, within 4 cognitive domains (memory, attention/executive function, language, and visuoconstruction):

- $\quad$ Rey Auditory Verbal Learning Test (RAVLT) [11] and Short Story Recall Test [12] for verbal memory. The RAVLT included immediate recall (the participants were given a list of 15 unrelated words repeated over 5 different trials) and uncued free delayed recall administered after $15 \mathrm{~min}$. In both tasks, the score was the total number of recalled words; higher scores represented better performance. The Short Story Recall Test was a 28-unit story used to measure both immediate and uncued free delayed recall (after 5 $\min$ ). The final score was the mean of the number of recalled items in both trials; higher scores represented better performance.

- Rey-Osterrieth Complex Figure [13] for constructional praxis (immediate copy: the participants were asked to copy a complex geometrical figure) and visuospatial memory (delayed recall: after $10 \mathrm{~min}$, the participants were asked to reproduce the figure from memory). In both tasks, the summary score was based on the presence and accuracy of 18 units of the figure (score range $0-36$ ); higher scores represented better performance.

- Trail Making Test [14] for psychomotor speed (part A, simple condition: the participants were asked to connect a sequence of 25 numbers in order) and divided attention (part B, alternating condition: the participants were asked to alternately connect a sequence of 25 numbers and letters). In both tasks, the score was the amount of time required to complete; higher scores represented worse performance.

- Visual search [15] for focalized attention. The task was to detect and cancel target digits within a time limit, and the score was the number of correct targets crossed out (score range 0-50); higher scores represented better performance.

- Symbol Digit Modalities Test [16] for maintained attention. The participants were asked to state the number that correctly corresponded to a symbol according to a key sequence. The score was the number of correct answers within a 90-s time period (score range 0-110); higher scores represented better performance. 


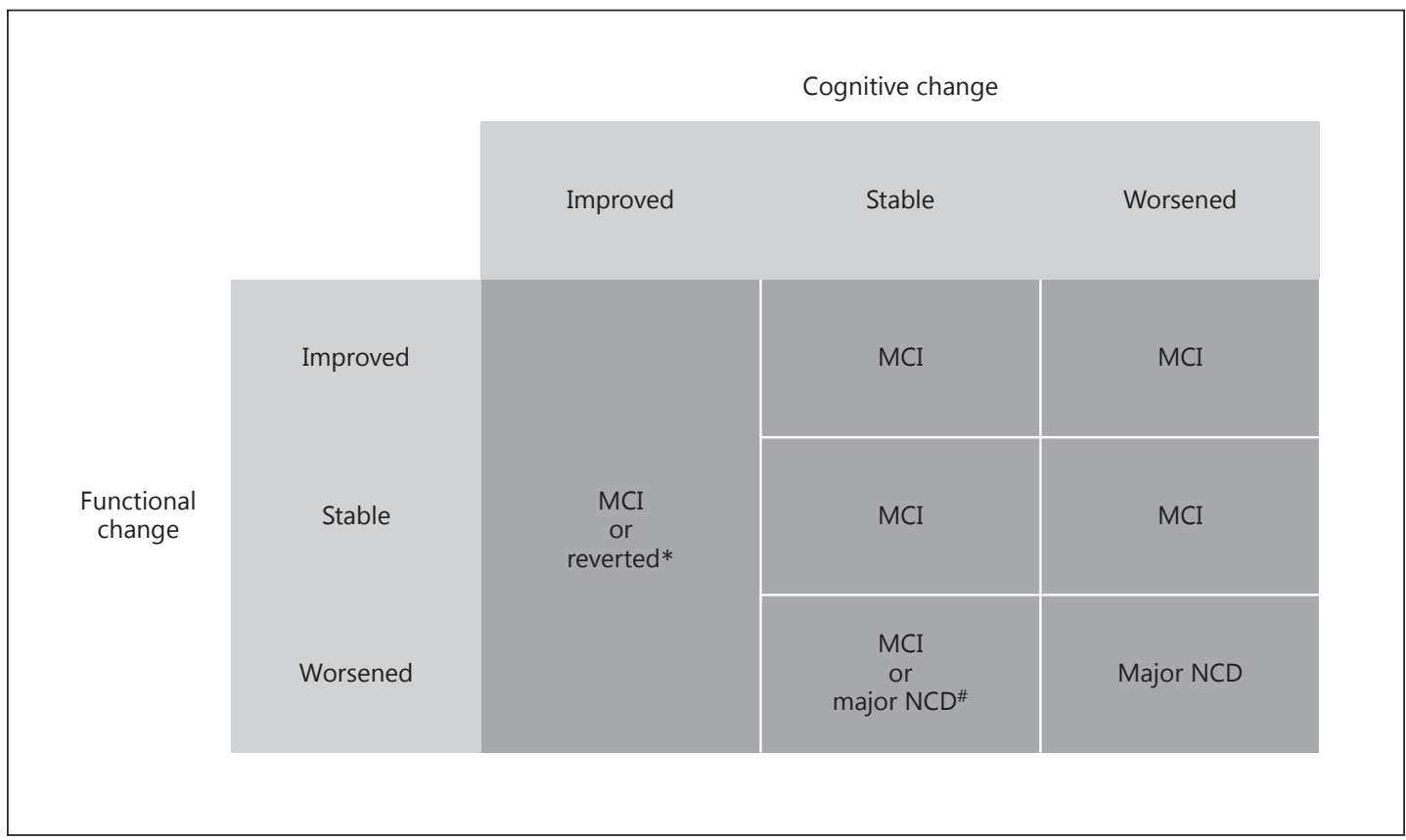

Fig. 1. Decision-making table for the determination of the final diagnosis. MCI, mild cognitive impairment; NCD, neurocognitive disorder. * If all cognitive tests resulted within the normal range. ${ }^{*}$ In case of a worsened functional outcome and a stable cognitive outcome, a major NCD diagnosis was established only if the functional status was not consequent to other diseases or physical limitations.

- Stroop Color-Word Test [17] for selective attention and response inhibition. In the congruous condition tasks, the participants were required to read color words printed in black ink and name different color patches, while in the incongruent condition task the participants were required to name the color of the ink of color words instead of reading. The interference effect was evaluated based on execution time (in seconds); higher scores represented worse performance.

- Phonemic (P-F-L) and semantic (animals-fruits-cars) verbal fluency tests [18] for language. For both tests, the final score was the total number of words produced for the 3 initials or categories, respectively; higher scores represented better performance.

The "worsened" cognitive condition was defined as reaching $\geq 1$ of 3 psychometric thresholds for change from baseline performance:

1. At least 1 additional impaired cognitive domain, i.e., $\geq 1$ impaired cognitive test result (performance $\geq 2$ SD or 3rd percentile below the norm) within a cognitive domain that was not impaired at the baseline evaluation

2. At least 1 additional impaired cognitive test result (performance $\geq 2$ SD or $3 r d$ percentile below the norm) within a cognitive domain that was already impaired at the baseline evaluation

3. At least 2 additional borderline cognitive test results (performance between 1 and 2 SD or 3rd-16th percentiles) in the presence of $\geq 1$ already impaired cognitive test result (performance $\geq 2$ SD or 3rd percentile below the norm)

The thresholds were conceived to represent 3 hierarchically ordered conditions of cognitive worsening, from the clinically most significant level of change ( 1 additional impaired cognitive domain) to the least significant one ( 2 additional borderline cognitive test results). 
Salvadori et al.: Application of Major NCD Criteria to MCl

Table 2. Comparison of baseline characteristics between patients that received a final diagnosis and dropouts

\begin{tabular}{lllrc}
\hline Baseline characteristic & $\begin{array}{l}\text { Score } \\
\text { range }\end{array}$ & $\begin{array}{l}\text { Patients with a final } \\
\text { diagnosis }(n=138)\end{array}$ & $\begin{array}{l}\text { Dropouts } \\
(n=15)\end{array}$ & $p$ \\
\hline Age, years & - & $74.4 \pm 6.8$ & $77.3 \pm 7.2$ & $0.131^{\mathrm{a}}$ \\
Years of education & - & $7.8 \pm 4$ & $8.9 \pm 5.5$ & $0.816^{\mathrm{a}}$ \\
Male & - & $78(56)$ & $6(40)$ & $0.222^{\mathrm{b}}$ \\
Mini-Mental State Examination score & $0-30$ & $27.4 \pm 2.7$ & $27.6 \pm 2.4$ & $0.918^{\mathrm{a}}$ \\
Montreal Cognitive Assessment score & $0-30$ & $21.6 \pm 4.6$ & $22.8 \pm 3.1$ & $0.380^{\mathrm{a}}$ \\
IADL scale score (impaired items) & $0-8$ & $0.8 \pm 1.4$ & $0.8 \pm 1.6$ & $0.688^{\mathrm{a}}$ \\
\hline
\end{tabular}

Values are means \pm SD or $n(\%)$. IADL, Instrumental Activities of Daily Living. ${ }^{\text {a }}$ Independent-sample nonparametric Mann-Whitney U test. ${ }^{\mathrm{b}} \chi^{2}$ test.

To evaluate the impact of including progressively less severe thresholds for cognitive worsening on the rate of patients diagnosed with major NCD, the 3 psychometric thresholds were applied successively in the abovementioned hierarchical order.

The intersection of the categorized cognitive and functional outcomes resulted in a $3 \times 3$ decision-making table that guided the determination of the final diagnosis (reverted, stable MCI, or major NCD) (Fig. 1). In case of a worsened functional outcome and a stable cognitive outcome, a major NCD diagnosis was established only if the functional status was not consequent to other diseases (e.g., stroke, heart diseases, etc.) or physical limitations. According to the DSM- 5 criteria for specification of the severity of major NCDs, patients were further classified as "mild" or "moderate" depending on whether they had difficulties in only instrumental or also basic activities of daily living. To make the final diagnosis, we considered the last evaluation (at 24 months). In case of an incomplete evaluation at 24 months or transition to major NCD at the first follow-up visit, we used data from the visit at 12 months.

\section{Statistical Analysis}

Independent-sample nonparametric Mann-Whitney $U$ tests and $\chi^{2}$ tests were used to compare patients that received the final diagnosis and dropouts according to baseline demographics (age, years of education, and sex), global cognitive functioning (adjusted scores on the Montreal Cognitive Assessment and Mini-Mental State Examination), and functional status (number of impaired items on the IADL scale).

Descriptive statistics were used to show the frequency distributions of longitudinal diagnostic categories. To verify whether the longitudinal diagnostic groups differed in baseline characteristics, independent-sample nonparametric Kruskal-Wallis and $\chi^{2}$ tests were used to compare demographics (age, years of education, and sex), global cognitive functioning (adjusted scores on the Montreal Cognitive Assessment and Mini-Mental State Examination), and functional status (number of impaired items on the IADL scale).

Univariate statistical analysis (Pearson's $\chi^{2}$ test) was used to compare the distributions of the baseline MCI subtypes across the longitudinal diagnostic categories.

\section{Results}

After a median follow-up of 24 months (interquartile range 15-25), 138 of the 153 patients of the VMCI-Tuscany baseline cohort (mean age $74.4 \pm 6.9$ years; male $57 \%$ ) had follow-up information sufficient to formulate a final diagnosis. Of the 15 patients that did not receive a 


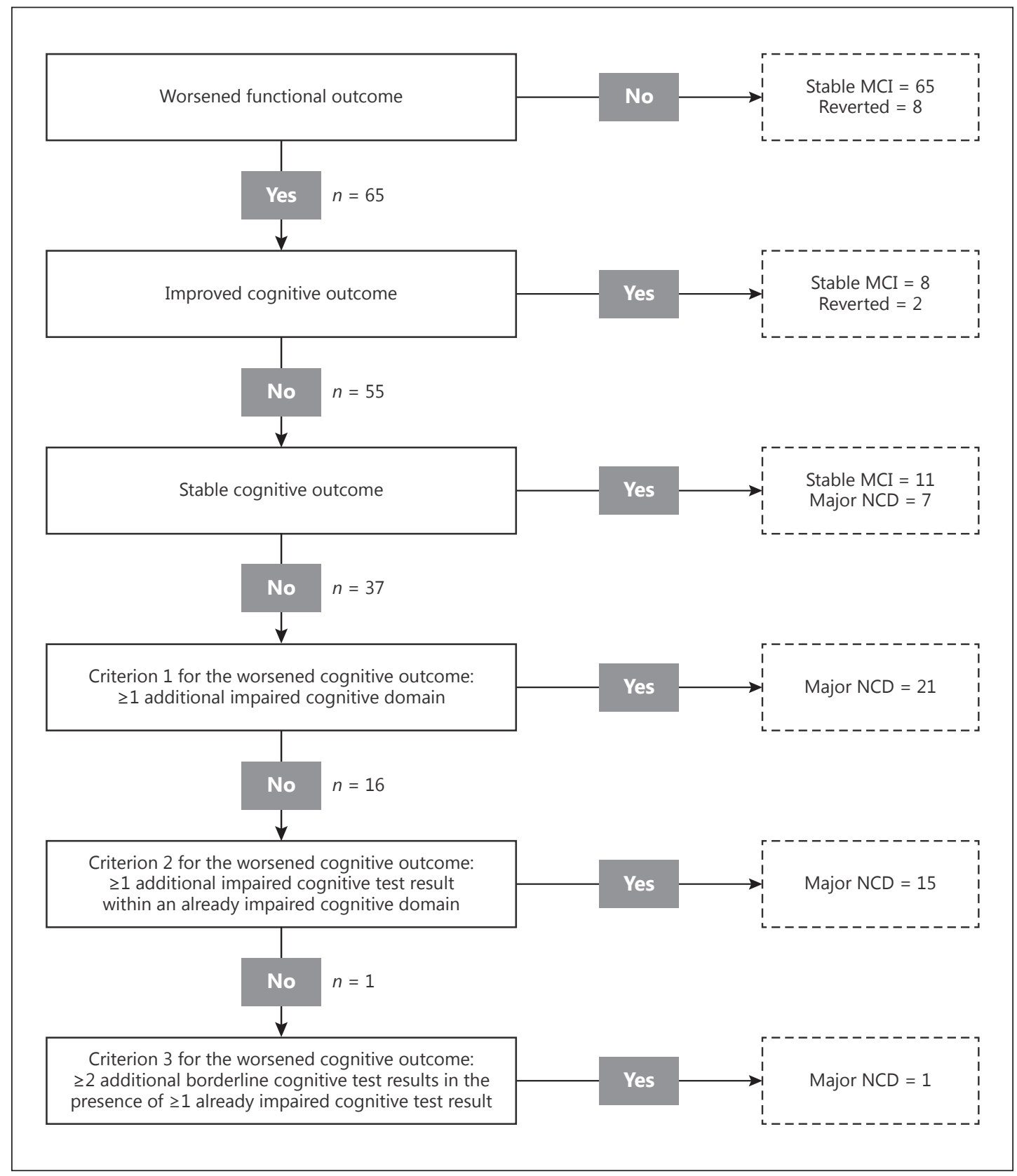

Fig. 2. Distribution of the patients' final diagnoses throughout the operationalized diagnostic algorithm. MCI, mild cognitive impairment; NCD, neurocognitive disorder.

diagnosis, 12 refused to undergo any follow-up visit and 3 underwent at least one follow-up visit but had incomplete evaluations. Comparisons between the 138 patients that received a diagnosis and the 15 excluded ones showed that there were no statistically significant differences in baseline demographics, global cognitive functioning, and functional status (Table 2).

Concerning the vascular risk factor distribution among the 138 patients of the final cohort, $115(83 \%)$ had hypertension, 86 (62\%) had hypercholesterolemia, $20(14 \%)$ had diabetes, 63 (46\%) had a smoking habit, 51 (37\%) had a history of stroke, and 43 (31\%) consumed alcohol. 
Table 3. Comparison of baseline characteristics between the diagnostic groups at follow-up

\begin{tabular}{llllll}
\hline Baseline characteristic & $\begin{array}{l}\text { Score } \\
\text { range }\end{array}$ & $\begin{array}{l}\text { Reverted to } \\
\text { normal } \\
\text { cognition } \\
(n=10)\end{array}$ & $\begin{array}{l}\text { Stable } \\
\text { MCI } \\
(n=84)\end{array}$ & $\begin{array}{l}\text { Major NCD } \\
(n=44)\end{array}$ & $p$ \\
& - & $73.8 \pm 9$ & $74.2 \pm 6.5$ & $75.1 \pm 6.9$ & $0.478^{\mathrm{a}}$ \\
Age, years & - & $8.6 \pm 2$ & $7.8 \pm 4.1$ & $7.5 \pm 4.2$ & $0.248^{\mathrm{a}}$ \\
Years of education & - & $4(40)$ & $48(57)$ & $26(59)$ & $0.537^{\mathrm{b}}$ \\
Male & $0-30$ & $29.1 \pm 0.8$ & $27.8 \pm 2.4$ & $26.3 \pm 3$ & $0.002^{\mathrm{a}}$ \\
Mini-Mental State Examination score & $0-30$ & $25.8 \pm 2.5$ & $21.8 \pm 4.3$ & $20.5 \pm 5$ & $0.002^{\mathrm{a}}$ \\
Montreal Cognitive Assessment score & $0-8$ & $0.1 \pm 0.3$ & $0.8 \pm 1.5$ & $0.9 \pm 1.3$ & $0.014^{\mathrm{a}}$ \\
IADL scale score (impaired items) & & & & & \\
\hline
\end{tabular}

Values are means \pm SD or $n(\%)$. MCI, mild cognitive impairment; NCD, neurocognitive disorder; IADL, Instrumental Activities of Daily Living. ${ }^{a}$ Independent-sample nonparametric Kruskal-Wallis test. ${ }^{\mathrm{b}} \chi^{2}$ test.

Of these 138 patients, 37 received their last evaluation at 12 months (18 due to an incomplete evaluation at 2 years and 19 due to transition to major NCD) and 101 at 24 months. Among the 138 patients with a final diagnosis, 10 (7\%) reverted to normal cognition, 84 (61\%) remained with $\mathrm{MCI}$, and $44(32 \%)$ had a diagnosis of major NCD (35 mild and 9 moderate) (Fig. 2).

Among the 44 patients diagnosed with major NCD, 7 (16\%) presented a worsened functional outcome and a stable cognitive outcome. All these patients had $\geq 1$ impaired cognitive domain, thus fulfilling the diagnostic criteria for major NCD. Twenty-one major NCD patients (48\%) presented $\geq 1$ additional impaired cognitive domain. Of the remaining 16 patients, 15 (34\%) had $\geq 1$ additional impaired cognitive test result in an already impaired cognitive domain, and 1 patient ( $2 \%$ ) fulfilled only the criterion of $\geq 2$ additional borderline cognitive test results. The results of the operationalization of the criteria for major NCD are shown in Figure 2.

Comparisons of the baseline characteristics between the longitudinal diagnostic groups are shown in Table 3. While there were no statistically significant differences in baseline demographics, the patients who received a diagnosis of major NCD had both significantly lower scores on the global cognitive functioning tests and a significantly higher number of impaired items on the IADL at the baseline evaluation.

The distribution of the 4 baseline MCI subtypes (amnestic - single or multiple domain; nonamnestic - single or multiple domain) across the longitudinal diagnostic groups is shown in Table 4 , and the $\chi^{2}$ analysis showed a significant association $(p<0.001)$. Half of the amnestic single-domain MCI patients $(n=4 ; 50 \%)$ reverted to normal cognition and the other half remained stable. Most of the nonamnestic single-domain MCI patients $(n=7 ; 70 \%)$ remained with $\mathrm{MCI}$, and the remaining $(n=3 ; 30 \%)$ reverted to normal cognition. Overall, single-domain MCI patients, mainly of the amnestic type, were more likely to revert to normal cognition than multiple-domain MCI patients (39 vs. 2.5\%), and no one received a diagnosis of major NCD. Considering multiple-domain MCI, most of the nonamnestic patients remained with MCI $(n=13 ; 72 \%), 3$ patients (17\%) received a diagnosis of major NCD, and $2(11 \%)$ reverted to normal cognition, while the amnestic subgroup had the highest proportion of patients that received a diagnosis of major NCD $(n=41 ; 40 \%)$ compared to all the other subgroups. 
Table 4. Distribution of baseline MCI subtypes across the longitudinal diagnostic groups

\begin{tabular}{llrrr}
\hline Baseline MCI subtype & \multicolumn{2}{l}{ Longitudinal diagnosis } & \multirow{2}{*}{ Total } \\
\cline { 2 - 4 } & $\begin{array}{l}\text { Reverted to } \\
\text { normal cognition }\end{array}$ & Stable MCI & Major NCD & \\
\hline Amnestic single domain & 4 & 4 & 0 & 8 \\
Amnestic multiple domain & 1 & 60 & 41 & 102 \\
Nonamnestic single domain & 3 & 7 & 0 & 10 \\
Nonamnestic multiple domain & 2 & 13 & 3 & 18 \\
\hline Total & 10 & 84 & 44 & 138 \\
\hline
\end{tabular}

MCI, mild cognitive impairment; NCD, neurocognitive disorder. $\chi^{2}$ test $p<0.001$.

\section{Discussion}

We proposed an operationalization of the DSM- 5 criteria for the determination of transition to major NCD in a longitudinal study. The DSM-5 criteria for major NCD require a significant cognitive decline (performance in formal testing 2 SD or 3rd percentile below the norm) from a previous level of performance in $\geq 1$ cognitive domain. Usually, the application of these criteria is cross-sectional and based on the psychometric distance between normative data and the patient performance measured at a single time point, or on a presumed or referred change from a previous level, and not on an objective evaluation of changes in the performance of the same patient on the same tests evaluated at two different time points. To the best of our knowledge, only one study applied an operationalization of the DSM-5 major NCD criteria to a population-based cohort - together with a cross-sectional assessment of decline also including an evaluation of the mean decline in cognitive test performance over time [19]. In our operationalization, the transition from mild to major NCD was entirely based on cognitive and functional changes from the baseline evaluation. For this, we defined hierarchically ordered psychometric thresholds that could represent a clinically significant change.

In our study, both criteria 1 and 2 (i.e., $\geq 1$ additional impaired cognitive test result either in an already impaired or a new cognitive domain) are an operationalization of the DSM-5 rules that define a decline in the same cognitive test severe enough to reach the psychometric threshold of $\geq 2$ SD or 3rd percentile below the norm. Eighty-two percent of our patients who received a major NCD diagnosis fulfilled criterion 1 or 2 , and most of them presented with impairment in a cognitive domain that had been normal at baseline. The addition of the less stringent criterion (i.e., $\geq 2$ additional borderline cognitive test results in the presence of $\geq 1$ already impaired cognitive test result) led to only 1 further patient being diagnosed with major NCD. Therefore, this criterion does not seem clinically significant.

Our research is in line with other emerging approaches to defining statistically derived neuropsychological criteria for the operational definition of subjective complaint, MCI, and dementia. As shown in several studies by Bondi and colleagues, the application of a neuropsychological method for actuarial diagnostic decision-making provided a more accurate and better characterization of MCI than did the use of conventional criteria based on clinical judgment and a simple cognitive screening [20]. Bondi and colleagues tested diagnostic approaches based either on statistically based cutoff points or on empirically derived clusters based on patterns of performance $[21,22]$, and the present study is in line with the former line of research. Our approach to a statistical diagnostic algorithm fulfills the 
need for a comprehensive multidomain neuropsychological evaluation and the incorporation of functional information into the decision-making process. Differently from previous statistically derived approaches to the diagnosis of cognitive impairment, our proposed diagnostic algorithm for major NCD is focused on the definition of clinically significant psychometric thresholds for change from a previous level of objective performance, and is hierarchically conceived to give primary importance to the number of impaired cognitive domains and secondary importance to the degree of deviation of test performances from normative data.

Because the distinction between mild and major NCD still depends on the preservation of functional activities, only patients with a worsened functional outcome and a stable or worsened cognitive outcome received a diagnosis of major NCD, while a worsened cognitive outcome without functional decline was not considered a sufficient condition. Conversely, in case of a stable cognitive outcome and a worsened functional status, the occurrence of other diseases or physical limitations that could have impacted the functional status was excluded before establishing a diagnosis of major NCD. In this case, we decided to still provide a diagnosis of major NCD, considering the functional decline as a marker of loss of the ability to use compensatory strategies in everyday activities due to a subthreshold cognitive decline or a failure of protective factors such as cognitive reserve. These patients could therefore represent a borderline category along the continuum from mild to major NCD, for which a worsened functional status could be considered as a stand-alone diagnostic criterion when a high degree of cognitive impairment is already evident and any other cause of the functional decline can be excluded.

In our cohort of MCI patients with SVD, about one-third progressed to major NCD after the 2-year follow-up, with few patients reverting to normal cognition. Only a few studies have evaluated the natural history of MCI patients with cerebral SVD or defined as having VMCI. Overall, the available evidence highlights some variability in conversion rates from MCI to dementia, ranging from about 15 to 50\% [23-28]. Several factors, such as the follow-up duration (ranging from 2 to 5 years) and the diagnostic criteria [23-28], likely explain this variability. The conversion rate in our short-term follow-up study was similar to that found in other studies with similar follow-up durations [24, 27, 28].

We also showed that patients with the multiple-domain MCI subtype (mainly the amnestic subgroup) are more likely to progress to major NCD, while those with the single-domain MCI subtypes have a less predictable outcome, with the highest proportion having reverted to normal cognition and no one having received a diagnosis of major NCD.

The hypothesis that different MCI subtypes could underlie different etiologies, and thus be useful in predicting specific dementia types, has important clinical implications in terms of preventive and therapeutic strategies [29]. The predictive accuracy of MCI subtypes for specific types of dementia is still somehow unclear [30-37], and beyond the aims of the present study. Taking into account conversion to all types of dementia, most studies report that patients with multiple-domain MCI have the highest risk of developing dementia, while patients with single-domain MCI have the highest frequency of reversion to normal cognition [30-32, 36, 37]. Thus, multiple-domain MCI could denote a greater extent of cognitive dysfunction than single-domain $\mathrm{MCI}$, and thereby represent a more advanced stage of disease $[38,39]$. On the other hand, single-domain MCI seems to have a more favorable prognosis, and could correspond to a heterogeneous category including patients in an early stage or cognitively normal individuals misdiagnosed as having MCI due to normal variability in cognitive test performances $[38,39]$. Our results are also in line with the hypothesis that MCI of presumed vascular etiology is mainly associated with impairment in multiple domains [29]. 
Some limitations of our study need to be considered. A first one is that the exclusion of clinical or physical factors that could have reduced the functional status of patients with a stable cognitive outcome was based on a certain set of information. Despite the great amount of available data, we cannot completely exclude the presence of other unknown or subtle clinical conditions.

A second possible limitation is that in our cohort the multiple-domain MCI groups were notably larger than the single-domain MCI groups, and this reduced the statistical power of the comparative analyses of the distribution of baseline MCI subtypes across the longitudinal diagnostic groups. Furthermore, the very limited sample sizes of the single-domain MCI groups decrease the generalizability of the evidence concerning their longitudinal outcomes, and our results need to be taken with caution and further explored in larger samples of MCI patients.

A third possible limitation concerns some shortcomings of the selected neuropsychological tests. The verbal memory tests of the VMCI-Tuscany neuropsychological battery included uncued free recall, which did not allow describing any specific profiles of the memory deficits, i.e., distinguishing between pure amnestic hippocampal and dysexecutive recall deficits. This choice was based on the unavailability of national normative studies of tests of verbal memory with cued recalls at the time the neuropsychological battery was developed. Another possible concern pertains to the ecological validity of the selected neuropsychological tests, which becomes of great relevance when neuropsychological testing has a key role in uncovering whether a patient's functional problems are a result of cognitive deficits. In line with evidence for the major part of traditional neuropsychological tools, the literature investigating the ecological validity of the neuropsychological tests used in the present study has shown conflicting results, and the magnitude of the significance of associations with measures of everyday skills was in the moderate range. To increase its ecological validity, neuropsychological assessment would benefit from the inclusion (among traditional test batteries) both of behavioral observations, rating scales, and self-report measures of functional status and of newer tests developed to maximize verisimilitude.

A last possible limitation is the lack of advanced imaging or biological markers for the identification of the etiology of NCDs. However, this reflects the situation in clinical settings, where there is limited access to advanced biomarkers. On the other hand, in real clinical practice several follow-up evaluations of the same patient by means of the same cognitive tests might not always be feasible, and this could further reduce the feasibility of the proposed approach. The present study aimed to propose one decision-making procedure for the diagnosis of major NCDs based on explicit psychometric thresholds, and this approach could be particularly useful in research settings and clinical services where patients undergo multiple functional and cognitive assessments over time.

In conclusion, we performed an operationalization of the DSM- 5 criteria for major NCDs in patients with SVD. According to this operationalization, we provided a rate of transition for this patient population to be used in future clinical trials and ongoing longitudinal studies.

\section{Acknowledgments}

We thank Prof. Perminder Sachdev (Centre for Healthy Brain Ageing, University of New South Wales, Sydney, NSW, Australia) for helpful revision of and comments on the manuscript during its early phase. 
Salvadori et al.: Application of Major NCD Criteria to MCl

\section{Funding Sources}

The VMCI-Tuscany study was funded by the Tuscany region. E.S. and R.V. were supported by a project funded by the Tuscany region and the Health Ministry under Grant Aimed Research Call 2010 (Bando Ricerca Finalizzata 2010; grant No. RF-2010-2321706; ClinicalTrials.gov identifier: NCT02033850).

The publication costs were funded by the nonprofit organization Associazione per la Ricerca sulle Demenze (ARD) ONLUS, Department of Neurology, Luigi Sacco Hospital, Milan, Italy.

\section{Disclosure Statement}

D.I. received research grants and a fee for conferences from SHIRE. M.C. received speaker honoraria from Biogen. N.D.S. serves on the scientific advisory boards for Merck Serono, Teva, Sanofi, Genzyme, Roche, Biogen, and Novartis Pharma AG; received travel funding from Biogen Idec, Merck Serono S.A., Bayer Schering AG, Teva, Sanofi, Genzyme, Roche, Biogen, and Novartis Pharma AG; is an editorial advisory board member for Neurological Sciences; and received honoraria from Biogen Idec, Merck Serono S.A., Bayer Schering AG, Teva, Sanofi, Genzyme, Roche, Biogen, and Novartis Pharma AG. E.S., A.P., G.P., A.C., L.C., A.D.B., S.D., I.D.D., A.G., S.M., M.M., S.N., G.O., M.P., F.P., R.V., A.F., M.T.D., U.B., and L.P. report no disclosures.

\section{References}

$>1$ O'Brien JT, Erkinjuntti T, Reisberg B, Roman G, Sawada T, Pantoni L, Bowler JV, Ballard C, DeCarli C, Gorelick PB, Rockwood K, Burns A, Gauthier S, DeKosky ST: Vascular cognitive impairment. Lancet Neurol 2003;2:89-98.

-2 Sachdev P, Kalaria R, O’Brien J, Skoog I, Alladi S, Black SE, Blacker D, Blazer DG, Chen C, Chui H, Ganguli M, Jellinger K, Jeste DV, Pasquier F, Paulsen J, Prins N, Rockwood K, Roman G, Scheltens P: Diagnostic criteria for vascular cognitive disorders: a VASCOG statement. Alzheimer Dis Assoc Disord 2014;28:206-218.

3 American Psychiatric Association: Diagnostic and Statistical Manual of Mental Disorders, ed 5. Arlington, American Psychiatric Publishing, 2013.

4 Poggesi A, Salvadori E, Pantoni L, Pracucci G, Cesari F, Chiti A, Ciolli L, Cosottini M, Del Bene A, De Stefano N, Diciotti S, Dotti MT, Ginestroni A, Giusti B, Gori AM, Nannucci S, Orlandi G, Pescini F, Valenti R, Abbate R, Federico A, Mascalchi M, Murri L, Inzitari D: Risk and determinants of dementia in patients with mild cognitive impairment and brain subcortical vascular changes: a study of clinical, neuroimaging, and biological markers - the VMCI-Tuscany study: rationale, design, and methodology. Int J Alzheimers Dis 2012;2012:608013.

-5 Winblad B, Palmer K, Kivipelto M, Jelic V, Fratiglioni L, Wahlund LO, Nordberg A, Bäckman L, Albert M, Almkvist O, Arai H, Basun H, Blennow K, de Leon M, DeCarli C, Erkinjuntti T, Giacobini E, Graff C, Hardy J, Jack C, Jorm A, Ritchie K, van Duijn C, Visser P, Petersen RC: Mild cognitive impairment - beyond controversies, towards a consensus: report of the International Working Group on Mild Cognitive Impairment. J Intern Med 2004;256: 240-246.

-6 Salvadori E, Poggesi A, Valenti R, Pracucci G, Pescini F, Pasi M, Nannucci S, Marini S, Del Bene A, Ciolli L, Ginestroni A, Diciotti S, Orlandi G, Di Donato I, De Stefano N, Cosottini M, Chiti A, Federico A, Dotti MT, Bonuccelli U, Inzitari D, Pantoni L: Operationalizing MCI criteria in small vessel disease: the VMCI-Tuscany Study. Alzheimers Dement 2016;12:407-418.

7 Pantoni L, Basile AM, Pracucci G, Asplund K, Bogousslavsky J, Chabriat H, Erkinjuntti T, Fazekas F, Ferro JM, Hennerici M, O’Brien J, Scheltens P, Visser MC, Wahlund LO, Waldemar G, Wallin A, Inzitari D: Impact of agerelated cerebral white matter changes on the transition to disability - the LADIS study: rationale, design and methodology. Neuroepidemiology 2005;24:51-62.

8 Salvadori E, Poggesi A, Pracucci G, Inzitari D, Pantoni L: Development and psychometric properties of a neuropsychological battery for mild cognitive impairment with small vessel disease: the VMCI-Tuscany Study. J Alzheimers Dis 2015;43:1313-1323.

-9 Katz S, Ford AB, Moskowitz RW, Jackson BA, Jaffe MW: Studies of illness in the aged. The index of ADL: a standardized measure of biological and psychosocial function. JAMA 1963;185:914-919.

10 Lawton MP, Brody EM: Assessment of older people: self-maintaining and instrumental activities of daily living. Gerontologist 1969;9:179-186.

11 Carlesimo GA, Caltagirone C, Gainotti G: The Mental Deterioration Battery: normative data, diagnostic reliability and qualitative analyses of cognitive impairment. The Group for the Standardization of the Mental Deterioration Battery. Eur Neurol 1996;36:378-384. 
Novelli G, Papagno C, Capitani E, Laiacona M, Cappa SF, Vallar G: Tre test clinici di memoria verbale a lungo termine. Taratura su soggetti normali. Arch Psicol Neurol Psichiatr 1986;47:278-296.

13 Caffarra P, Vezzadini G, Dieci F, Zonato F, Venneri A: Rey-Osterrieth complex figure: normative values in an Italian population sample. Neurol Sci 2002;22:443-447.

14 Giovagnoli AR, Del Pesce M, Mascheroni S, Simoncelli M, Laiacona M, Capitani E: Trail Making Test: normative values from 287 normal adult controls. Ital J Neurol Sci 1996;17:305-309.

15 Della Sala S, Laiacona M, Spinnler H, Ubezio C: A cancellation test: its reliability in assessing attentional deficits in Alzheimer's disease. Psychol Med 1992;22:885-901.

-16 Nocentini U, Giordano A, Di Vincenzo S, Panella M, Pasqualetti P: The Symbol Digit Modalities Test - oral version: Italian normative data. Funct Neurol 2006;21:93-96.

17 Caffarra P, Vezzadini G, Dieci F, Zonato F, Venneri A: Una versione abbreviata del test di Stroop. Dati normativi nella popolazione italiana. Nuova Riv Neurol 2002;12:111-115.

18 Novelli G, Papagno C, Capitani E, Laiacona M, Vallar G: Tre test clinici di ricerca e produzione lessicale: taratura su soggetti normali. Arch Psicol Neurol Psichiatr 1986;47:477-506.

19 Eramudugolla R, Mortby ME, Sachdev P, Meslin C, Kumar R, Anstey KJ: Evaluation of a research diagnostic algorithm for DSM-5 neurocognitive disorders in a population-based cohort of older adults. Alzheimers Res Ther 2017;9:15.

20 Bondi MW, Smith GE: Mild cognitive impairment: a concept and diagnostic entity in need of input from neuropsychology. J Int Neuropsychol Soc 2014;20:129-134.

21 Jak AJ, Bondi MW, Delano-Wood L, Wierenga C, Corey-Bloom J, Salmon DP, Delis DC: Quantification of five neuropsychological approaches to defining mild cognitive impairment. Geriatr Psychiatry 2009;17:368-375.

-22 Clark LR, Delano-Wood L, Libon DJ, McDonald CR, Nation DA, Bangen KJ, Jak AJ, Au R, Salmon DP, Bondi MW: Are empirically-derived subtypes of mild cognitive impairment consistent with conventional subtypes? J Int Neuropsychol Soc 2013;19:635-645.

23 Wentzel C, Rockwood K, MacKnight C, Hachinski V, Hogan DB, Feldman H, Østbye T, Wolfson C, Gauthier S, Verreault R, McDowell I: Progression of impairment in patients with vascular cognitive impairment without dementia. Neurology 2001;57:714-716.

-24 Luis CA, Barker WW, Loewenstein DA, Crum TA, Rogaeva E, Kawarai T, St George-Hyslop P, Duara R: Conversion to dementia among two groups with cognitive impairment. A preliminary report. Dement Geriatr Cogn Disord 2004;18:307-313.

25 Bombois S, Debette S, Bruandet A, Delbeuck X, Delmaire C, Leys D, Pasquier F: Vascular subcortical hyperintensities predict conversion to vascular and mixed dementia in MCI patients. Stroke 2008;39:2046-2051.

-26 Marra C, Ferraccioli M, Vita MG, Quaranta D, Gainotti G: Patterns of cognitive decline and rates of conversion to dementia in patients with degenerative and vascular forms of MCI. Curr Alzheimer Res 2011;8:24-31.

-27 Clerici F, Caracciolo B, Cova I, Fusari Imperatori S, Maggiore L, Galimberti D, Scarpini E, Mariani C, Fratiglioni L: Does vascular burden contribute to the progression of mild cognitive impairment to dementia? Dement Geriatr Cogn Disord 2012;34:235-243.

28 Kim S, Choi SH, Lee YM, Kim MJ, Kim YD, Kim JY, Park JH, Myung W, Na HR, Han HJ, Shim YS, Kim JH, Yoon SJ, Kim SY, Kim DK: Periventricular white matter hyperintensities and the risk of dementia: a CREDOS study. Int Psychogeriatr 2015;27:2069-2077.

29 Gauthier S, Reisberg B, Zaudig M, Petersen RC, Ritchie K, Broich K, Belleville S, Brodaty H, Bennett D, Chertkow H, Cummings JL, de Leon M, Feldman H, Ganguli M, Hampel H, Scheltens P, Tierney MC, Whitehouse P, Winblad B: Mild cognitive impairment. Lancet 2006;367:1262-1270.

-30 Alexopoulos P, Grimmer T, Perneczky R, Domes G, Kurz A: Progression to dementia in clinical subtypes of mild cognitive impairment. Dement Geriatr Cogn Disord 2006;22:27-34.

-31 Nordlund A, Rolstad S, Klang O, Edman A, Hansen S, Wallin A: Two-year outcome of MCI subtypes and aetiologies in the Göteborg MCI study. J Neurol Neurosurg Psychiatry 2010;81:541-546.

-32 Aerts L, Heffernan M, Kochan NA, Crawford JD, Draper B, Trollor JN, Sachdev PS, Brodaty H: Effects of MCI subtype and reversion on progression to dementia in a community sample. Neurology 2017;88:2225-2232.

33 Busse A, Hensel A, Gühne U, Angermeyer MC, Riedel-Heller SG: Mild cognitive impairment: long-term course of four clinical subtypes. Neurology 2006;67:2176-2185.

34 Yaffe K, Petersen RC, Lindquist K, Kramer J, Miller B: Subtype of mild cognitive impairment and progression to dementia and death. Dement Geriatr Cogn Disord 2006;22:312-319.

-35 Fischer P, Jungwirth S, Zehetmayer S, Weissgram S, Hönigschnabl S, Gelpi E, Krampla W, Tragl KH: Conversion from subtypes of mild cognitive impairment to Alzheimer dementia. Neurology 2007;68:288-291.

-36 Rasquin SM, Lodder J, Visser PJ, Lousberg R, Verhey FR: Predictive accuracy of MCI subtypes for Alzheimer's disease and vascular dementia in subjects with mild cognitive impairment: a 2-year follow-up study. Dement Geriatr Cogn Disord 2005;19:113-119.

37 Han JW, Kim TH, Lee SB, Park JH, Lee JJ, Huh Y, Park JE, Jhoo JH, Lee DY, Kim KW: Predictive validity and diagnostic stability of mild cognitive impairment subtypes. Alzheimers Dement 2012;8:553-559.

-38 Sachdev PS, Lipnicki DM, Crawford J, Reppermund S, Kochan NA, Trollor JN, Draper B, Slavin MJ, Kang K, Lux O, Mather KA, Brodaty H: Risk profiles of subtypes of mild cognitive impairment: the Sydney Memory and Ageing Study. J Am Geriatr Soc 2012;60:24-33.

-39 Roberts R, Knopman DS: Classification and epidemiology of MCI. Clin Geriatr Med 2013;29:753-772. 